Journal of Computer Science 6 (2): 194-198, 2010

ISSN 1549-3636

(C) 2010 Science Publications

\title{
Performance of Orthogonal Frequency Division Multiplexing System Based on Mobile Velocity and Subcarrier
}

\author{
${ }^{1}$ Zulkeflee Bin Khalidin and ${ }^{2}$ Haitham J. Taha \\ ${ }^{1}$ Faculty of Electrical Engineering, University of Malaysia Pahang, Kuantan, Malaysia \\ ${ }^{2}$ Faculty of Electrical and Electronic Engineering, University Sains Malaysia, Penang, Malaysia
}

\begin{abstract}
Problem statement: Nowadays, the mobile telecommunications industry faces the problem of providing the technology that be able to support a wide variety of services ranging from voice communication with a bit rate of a few kbps to wireless multimedia in which bit rate up to 2 Mbps. Approach: This study introduced a new performance study to enhance the Orthogonal Frequency Division Multiplexing (OFDM) system based on both efficient iterative number of subcarrier estimation techniques and a tracking algorithm was proposed. Result: The performance of system degrades with increase of the mobile velocity or increase of the number of channel fingers. The noiseless received signal at the weakest subcarrier is estimated based on all the detected data symbols using a hard or soft decision. Due to the flexibility of the transform design, the analysis conducted is applicable for other common systems as well. Conclusion: Simulation results showed the proposed algorithm can track the velocity variation very well and perform channel estimation using the most efficient tracking mode.
\end{abstract}

Key words: FFT, OFDM, WPT

\section{INTRODUCTION}

Nowadays, in wireless communication systems, the ability of OFDM to efficiently exploit the wideband properties of the radio channel lies at the heart of its popularity plays a very important role to combat the doubly dispersive channels. This feature has helped to establish OFDM as the physical layer of choice for broadband wireless communications systems (European Telecommunications Standards Institute, 2002; National Institute of Informatics, 2002; IEEE, 2003). Therefore, it is expected that OFDM will be still a fundamental element in future wireless communications.

Recently, a considerable amount of research work has been proposed as candidate for the 3rd generation mobile telecommunication system, OFDM is one of proposed system that has a lot of attentions for some reasons. The first reason is that the successful implementation of OFDM in several applications. OFDM has successfully been implemented in the wireless broadcasting applications such as Digital Video Broadcasting (DVB) and Digital Audio Broadcasting (DAB) (Martoyo and Jondral, 2002). Since OFDM can overcome the effect of multipath fading by using a guard period, OFDM need only a simple equalization method in combating selective fading channels (Litwin, 2000). Increasing symbol duration will result in lower rate parallel subcarriers. The transmitter and the receiver for OFDM system can be implemented efficiently by using Fast Fourier Transform (FFT) techniques (Martoyo and Jondral, 2002). Adding a Cyclic Prefix (CP) is the main way for the Fourier based OFDM to eliminate the ISI. However, this can decrease the bandwidth efficiency greatly, which means that there is a long way go to improve the bandwidth efficiency, to decrease the bandwidth waste brought by adding CP (Gupta et al., 2008). Past research (Dowler and Nix, 2003) has investigated the use of scattered pilots combined with various interpolation filters to achieve a channel estimate.

For OFDM to operate in a wide area network, efficient channel estimation techniques are required that operate in fast time varying channels. However, the time selective channel resulting from the relative movement is still a challenging task for channel estimation algorithms. In this study, we investigate the performance of the two major obstacles in using OFDM in transmission system; first it is very sensitive to frequency offset caused by misalignment in carrier frequencies or Doppler shift. These imperfections will destroy sub-carrier orthogonality and introduce Inter-

Corresponding Author: Zulkeflee Bin Khalidin, Faculty of Electrical Engineering, University of Malaysia Pahang, Kuantan, Malaysia 
Carrier Interference (ICI) among sub-carrier in addition to attenuation and rotation of each of the sub-carriers phase, second disadvantage is that the peak power of the signal can be up to $\mathrm{N}$ times the average power (where $\mathrm{N}$ is the number of carriers) (Sklar, 2001), to achieve the same optimum performance as in the case of preceding over all subcarriers, where is the number of channel taps. The simulation result shows the performance of system degrades with increase of the mobile velocity or increase of the number of channel fingers.

\section{MATERIALS AND METHODS}

FFT-OFDM system: The FFT-OFDM system model is shown in Fig. 1. The data is transmitted in the parallel by assigning each data word to one carrier in the transmission. The data to be transmitted on each carrier is modulated into a BPSK format. After the required spectrum is worked out, an IFFT is used to find the corresponding time domain waveform.

The cyclic prefix is then added to the start of each symbol. The receiver basically does the inverse operation to the transmitter. The cyclic prefix is removed. The FFT of each symbol is then taken to find the original transmitted spectrum. Single tap frequency domain equalizer is used to enhance the detection performance. Each transmitted carrier is then evaluated and converted back to the data word by demodulating the received symbol. The data words are then combined back to the same word size as the original signal.

Doppler shift: When a signal source and a receiver are moving related to one another, the frequency of received signal will not be the same as the source. Consider a mobile moving at a constant velocity $\mathrm{v}$, along a path segment having length $\mathrm{d}$ between points $\mathrm{X}$ and $Y$, while it receives signals from a remote source $S$, as illustrated in Fig. 2. The difference in path lengths traveled by the wave from source $S$ to the mobile at points $\mathrm{X}$ and $\mathrm{Y}$ is $\Delta \mathrm{l}=\mathrm{d} \cos \theta=\mathrm{v} \Delta \mathrm{t} \cos \theta$ where $\Delta \mathrm{t}$ is the time required for the mobile to travel from $\mathrm{X}$ to $\mathrm{Y}$ and $\theta$ is assumed to be the same at point $\mathrm{X}$ and $\mathrm{Y}$ since the source is assumed to be very far way. The phase change in the received signal due to difference in path lengths is therefore (Lee, 1995):

$\Delta \phi=\frac{2 \pi \Delta \mathrm{l}}{\lambda}=\frac{2 \pi \mathrm{v} \Delta \mathrm{t}}{\lambda} \cos \theta$

Hence the apparent change in frequency, or Doppler shift, is given by: $\mathrm{f}_{\mathrm{d}}=\frac{1}{2 \pi} \cdot \frac{\Delta \phi}{\Delta \mathrm{t}}=\frac{\mathrm{v}}{\lambda} \cos \theta$

It can be seen from Eq. 2 that if the mobile is moving toward the direction of arrival of the wave, the Doppler shift is positive and if the mobile is moving away from the direction of arrival of the wave, the Doppler shift is negative (Rappaport, 1996).

Parameters of mobile multipath channel: In order to compare different channels and to develop some general design guidelines for wireless systems, parameters which closely quantify the multipath channel are used. These parameters include delay spread and Doppler spread (Lee, 1995; Rappaport, 1996).

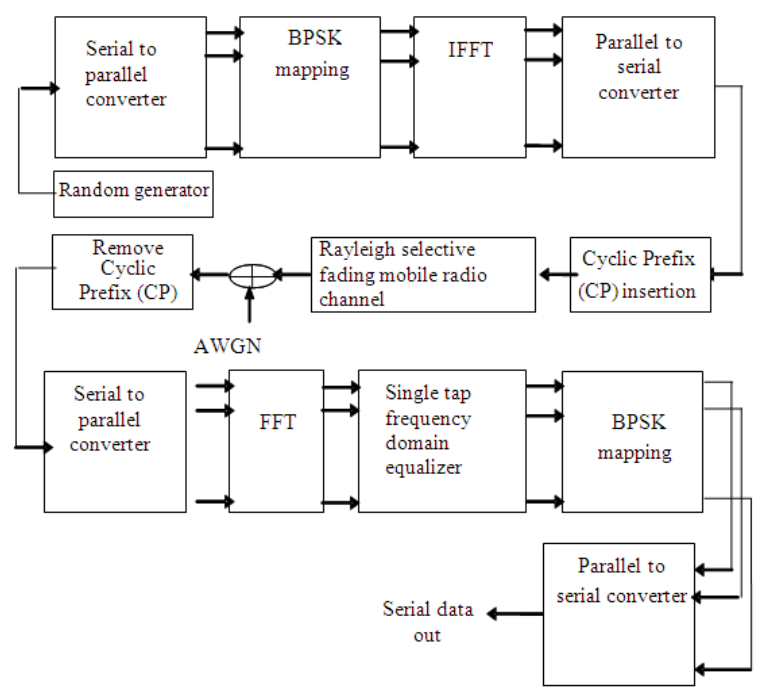

Fig. 1: FFT-OFDM model

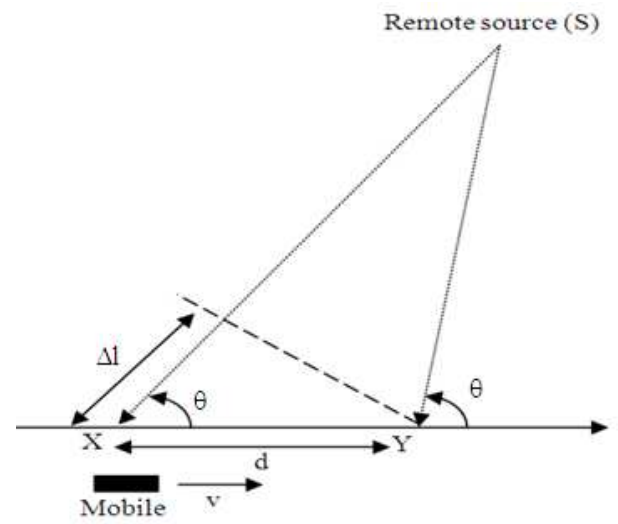

Fig. 2: Doppler shift 
Delay spread and coherence bandwidth: Delay spread and coherence bandwidths are parameters which describe the time dispersive nature of the channel in local area. The time dispersive properties of wide band multipath channels are most commonly quantified by their mean excess delay $(\tau)$ and RMS delay spread $\left(\sigma_{\tau}\right)$. The mean excess delay is given by:

$\bar{\tau}=\frac{\sum_{\mathrm{K}} \mathrm{a}_{\mathrm{K}}{ }^{2} \tau_{\mathrm{K}}}{\sum_{\mathrm{K}} \mathrm{a}_{\mathrm{K}}{ }^{2}}=\frac{\sum_{\mathrm{K}} \mathrm{P}\left(\tau_{\mathrm{K}}\right) \tau_{\mathrm{K}}}{\sum_{\mathrm{K}} \mathrm{P}\left(\tau_{\mathrm{K}}\right)}$

Where:

$\mathrm{P}(\tau)=$ The absolute power level of received signal

$\mathrm{a}^{2}=\mathrm{P}(\tau)$

The RMS delay spread is defined to be:

$\sigma_{\tau}=\sqrt{\overline{\tau^{2}}-(\bar{\tau})^{2}}$

Where:

$$
\overline{\tau^{2}}=\frac{\sum_{\mathrm{K}} \mathrm{a}_{\mathrm{K}}{ }^{2} \tau_{\mathrm{K}}{ }^{2}}{\sum_{\mathrm{K}} \mathrm{a}_{\mathrm{K}}{ }^{2}}=\frac{\sum_{\mathrm{K}} \mathrm{P}\left(\tau_{\mathrm{K}}\right) \tau_{\mathrm{K}}{ }^{2}}{\sum_{\mathrm{K}} \mathrm{P}\left(\tau_{\mathrm{K}}\right)}
$$

These delays are measured relative to the first detectable signal arriving at the receiver at $\tau_{\mathrm{o}}=0$.

The coherence bandwidth, $\mathrm{B}_{\mathrm{C}}$, can be defined as the relation derived from the RMS delay spread. If the coherence bandwidth is defined as the bandwidth over which the frequency correlation function is above 0.9, then the coherence bandwidth is approximately:

$$
\mathrm{B}_{\mathrm{C}} \approx \frac{1}{50 \sigma_{\tau}}
$$

If the definition is relaxed so that the frequency correlation function is above 0.5 , then the coherence bandwidth is approximately:

$$
\mathrm{B}_{\mathrm{C}} \approx \frac{1}{5 \sigma_{\tau}}
$$

Doppler spread and coherence time: Doppler spread and coherence time are parameters which describe the time varying nature of the channel in small scale region. Doppler spread, $\mathrm{B}_{\mathrm{D}}$, is a measure of the spectral broadening caused by the time rate of change of mobile radio channel. It can be defined as the range of frequencies over which the received Doppler spectrum is essentially non-zero. Coherence time, $\mathrm{T}_{\mathrm{C}}$, is the time domain dual of Doppler spread which can be used to characterize the time varying nature of the frequency depressiveness of the channel in the time domain. The Doppler spread and coherence time are inversely proportional to one another. That is:

$$
\mathrm{T}_{\mathrm{C}}=\frac{1}{\mathrm{~B}_{\mathrm{D}}}
$$

From the above, it can be seen that the time dispersion and frequency dispersion mechanisms in a mobile radio channel lead to four possible distinct effects, which are manifested depending on the nature of the transmitted signal, the channel and the velocity. The two propagation mechanisms are independent of one another.

\section{RESULTS}

The parameters and system configuration used in the simulation were taken from the standards of the third generation mobile and OFDM system. These parameters can be summarized by the followings:

$\begin{array}{ll}\text { Data rate } & 2 \mathrm{Mbps} \\ \text { Modulation } & \text { BPSK } \\ \text { Subcarriers } & 128 \\ \text { FFT points } & 128 \\ \text { DWT pints } & 256 \\ \text { OFDM symbol duration } & 16 \times 10^{-6} \mathrm{sec} \\ \text { Guard interval } & 1.6 \times 10^{-6} \mathrm{sec} \\ \text { Guard interval type } & \text { Cyclic Prefix (CP) } \\ \text { Required bandwidth } & 2 \mathrm{MHz} \\ \text { Model of channel } & \text { Jacks model } \\ \text { Number of paths } & 8 \text { path } \\ \text { Number of paths } & 2 \text {-fingers } \\ \text { Multipath delay spread } & 3 \times 10^{-6} \mathrm{sec} \\ \text { Doppler frequency } & 150 \mathrm{~Hz}\end{array}$

To investigate the effect of number of subcarrier on the OFDM system extra test was carried out as shown in Fig. 3. From the Fig. 3, the performance of system is enhanced as the number of subcarrier increased.

In these tests the selective fading configuration contains 2-fingers, 3-fingers, 4-fingers and so on, the effect of increasing number of finger on the performance of system is shown in Fig. 4. This increase in the number of fingers causes performance degradation. From the Fig. 4 it is clear that the performance of 2-fingers is better than the performance of 4 -fingers by about $2 \mathrm{~dB}$ at bit error rate $10^{-5}$. Also the performance of 4 -fingers better than the performance of 6 -fingers by about $4 \mathrm{~dB}$ at bit error rate $10^{-5}$. 


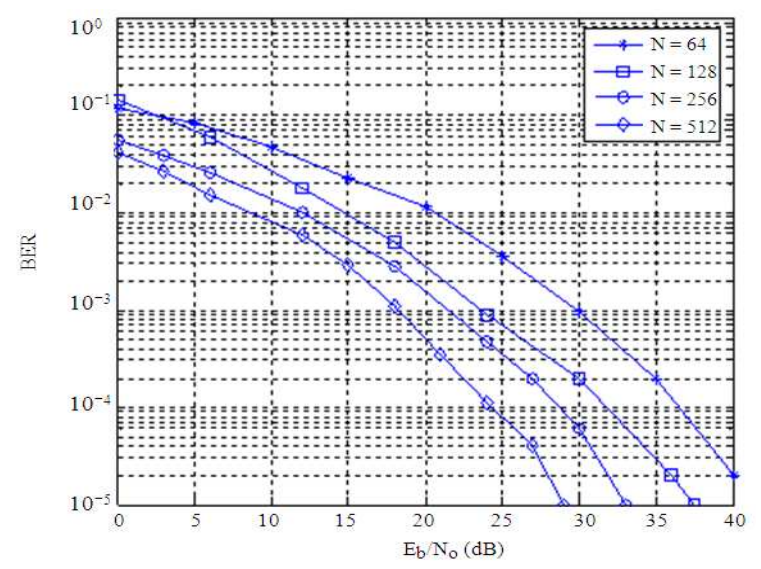

Fig. 3: FFT-OFDM system at different number of subcarrier

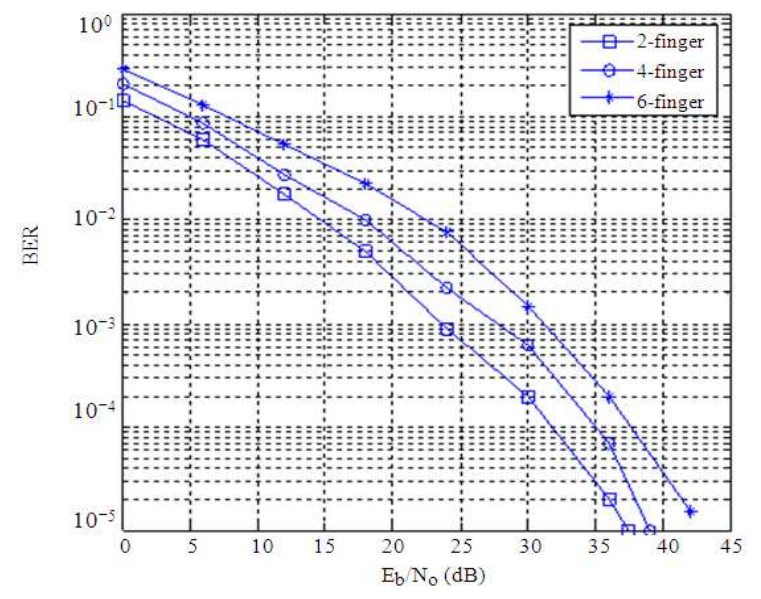

Fig. 4: FFT-OFDM system at different number of fingers

\section{DISCUSSION}

The relation between the number of subcarrier and energy per bit to noise ratio $\left(\mathrm{E}_{\mathrm{b}} / \mathrm{N}_{\mathrm{o}}\right)$ in $\mathrm{dB}$ at different number of fingers was shown in Fig. 5. The $E_{b} / N_{o}(d B)$ decreased as the number of subcarrier increased. This relation at bit error rate $10^{-5}$.

The performance of OFDM system with different mobile velocity $\left(\right.$ mile $\mathrm{h}^{-1}$ ) is shown in Fig. 6. The performance degrades with increased mobile velocity.

Figure 7 shows the effect of increasing the velocity of mobile station on the performance of OFDM system at different number of subcarrier. The Fig. 7 shows the performance degrade with increasing velocity. When the velocity increased the Doppler frequency increased and hence the number of fade $\mathrm{sec}^{-1}$ increased. Therefore, the performance degrades.

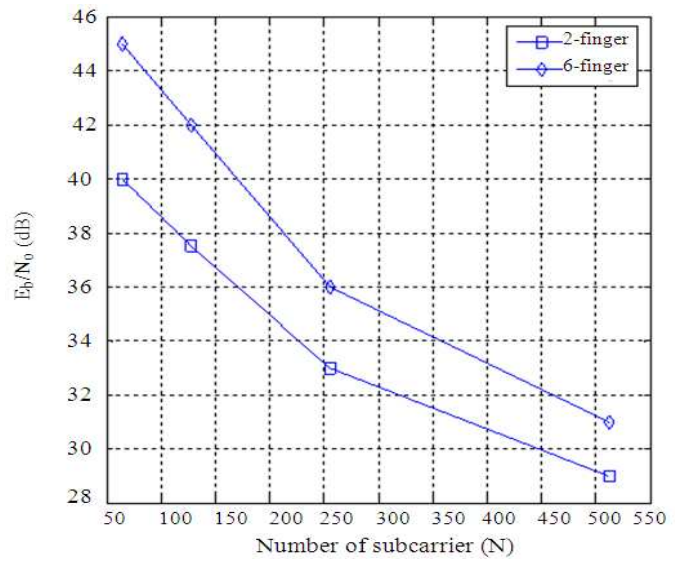

Fig. 5: Relation between number of subcarrier and $\mathrm{E}_{\mathrm{b}} / \mathrm{N}_{\mathrm{o}}$ (dB) at different number of selective channel fingers

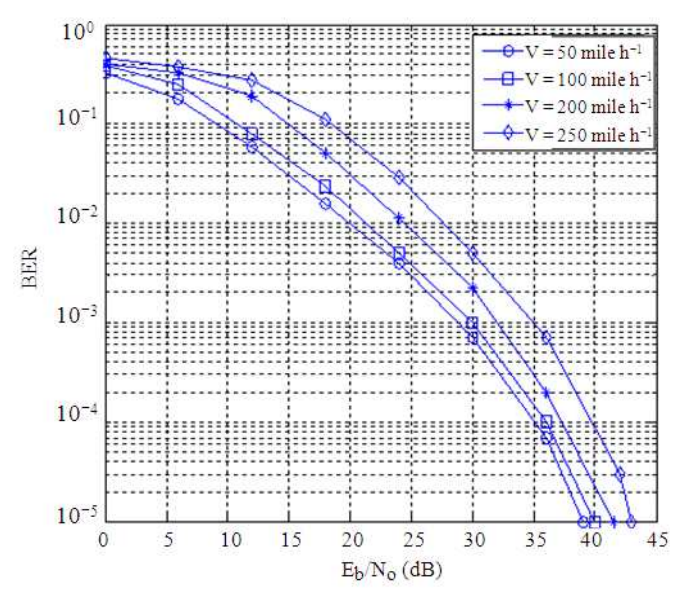

Fig. 6: FFT-OFDM system at different mobile velocity $\left(\right.$ mile $\mathrm{h}^{-1}$ )

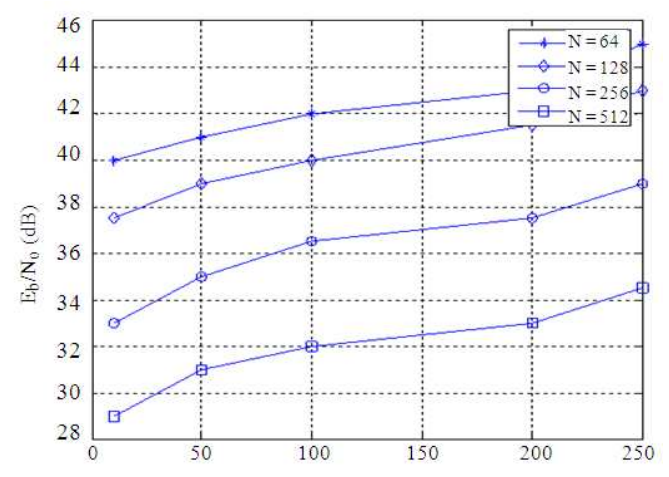

Fig. 7: Relation between mobile velocity and $E_{b} / N_{o}$ $(\mathrm{dB})$ in FFT-OFDM system at different number of subcarrier and number of finger $=2$ 


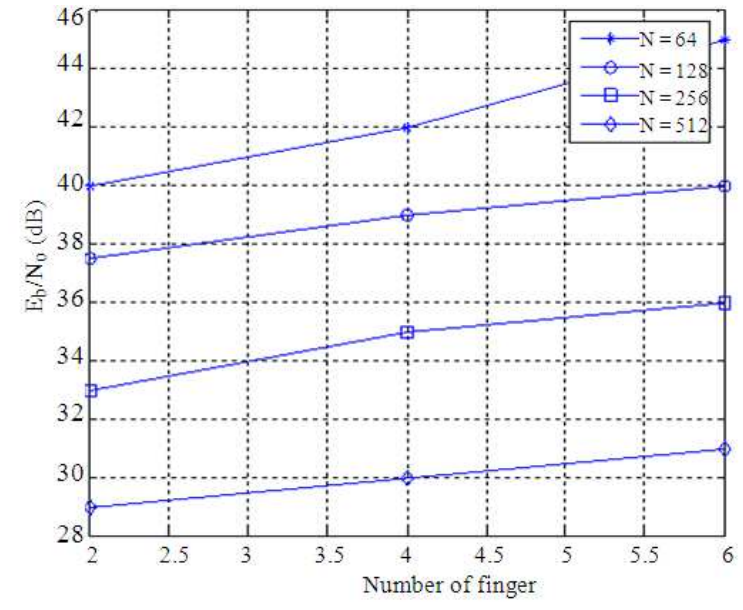

Fig. 8: Relation between number of finger and $E_{b} / N_{o}$ (dB) in FFT-OFDM system at different number of subcarrier and mobile velocity $=50 \mathrm{mile} \mathrm{h}^{-1}$

The relation between the number of finger and $\mathrm{E}_{\mathrm{b}} / \mathrm{N}_{\mathrm{o}}(\mathrm{dB})$ at bit error rate $10^{-5}$ was shown in Fig. 8. The best performance occurs with minimum number of finger. When the number increased the value of Inter Symbol Interference (ISI) increased. Therefore, the performance degraded.

\section{CONCLUSION}

OFDM is one of the main-stream multiplexing techniques for modern communication systems. Moreover, the channel estimation algorithm plays an important role for improving the system performance and computational complexity. This study focuses on performance of the Orthogonal Frequency Division Multiplexing (OFDM) system based on both efficient iterative number of subcarrier estimation techniques and a tracking algorithm. Firstly, it introduces the sensitiveness of OFDM to frequency offset caused by misalignment in carrier frequencies or Doppler shift and introduces Inter-Carrier Interference (ICI) among sub-carrier in addition to attenuation and rotation of each of the sub-carriers phase. Secondly, it discusses its disadvantage, that is, the peak power of the signal can be up to $\mathrm{N}$ times the average power. Finally, simulations in practical wireless channels demonstrate the superior performance of the iterative detector as compared to conventional detectors with comparable complexity, while obtain reasonable channel estimation outputs in slow-moving cases with low-complexity modes.

\section{REFERENCES}

Dowler, A. and A. Nix, 2003. Performance evaluation of channel estimation techniques for a mobile $4 \mathrm{G}$ wide area OFDM system. Proceeding of the IEEE Vehicular Technology Conference, Oct. 6-9, IEEE Xplore Press, USA., pp: 1214-1218.

IEEE., 2003. Part11: Wireless LAN Medium Access Control (MAC) and Physical Layer (PHY) specifications: High speed physical layer in the 5 $\mathrm{GHz}$ band.

http://www.citeulike.org/user/emedepan/article/174 6138

European Telecommunications Standards Institute, 2002. ETSI EN 301958 Digital Video Broadcasting (DVB); Interaction channel for Digital Terrestrial Television (RCT) incorporating Multiple Access OFDM. http://electronics.ihs.com/document/abstract/KMZ XTAAAAAAAAAAA

Gupta, D., V.B. Vats, and K.K. Garg, 2008. Performance analysis of DFT-OFDM, DCTOFDM, and DWT-OFDM systems in AWGN channel. Proceeding of the 4th International Conference on Wireless and Mobile Communications, July 27-Aug. 01, IEEE Computer Society, Washington DC., USA., pp: 214-216. DOI: 10.1109/ICWMC.2008.66

Lee, W., 1995. Mobile Cellular Telecommunications. Mc-Graw Hill, Inc., ISBN: 0-07-051726-6, pp: 276.

Litwin, L., 2000. An introduction to multicarrier modulation. IEEE Potent., 19: 36-38. DOI: $10.1109 / 45.839645$

Martoyo, H.S. and F. Jondral, 2002. CDMA versus OFDM, a performance comparison in selective fading channels. Proceeding of the IEEE Seventh International Symposium on Spread Spectrum Techniques and Applications, Sept. 2002, IEEE Xplore Press, Prague, Czech Republic, pp: 139143. DOI: $10.1109 /$ ISSSTA.2002.1049302

National Institute of Informatics, 2002. Broadband Radio Access Networks (BRAN); HIPERLAN type 2; Data Link Control (DLC) layer; Part 2: Radio Link Control (RLC) sublayer. http://ci.nii.ac.jp/naid/10012882167/en/

Rappaport, T., 1996. Wireless Communications Principles and Practice. Prentice Hall, ISBN: 10: 0130422320, pp: 736.

Sklar, B., 2001. Digital Communications Fundamentals and Applications. 2nd Edn., Prentice Hall, ISBN: 0-130-84788-7. 